\title{
Multi-Technique groundwater flow system analysis and dating of deep aquifers in Alessandria Basin (Piedmont - IT)
}

\section{Studio interdisciplinare e datazione del sistema acquifero profondo del bacino alessandrino (Piemonte, IT)}

\author{
Nicola Quaranta, Elena Cogo, Adriano Simoni, Elisa Sacchi, Mariachiara Caschetto, Massimo Marchesi
}

Riassunto: Lo scopo dello studio è stato quello di porre in essere un sistema di salvaguardia dalla contaminazione del sistema acquifero profondo del bacino di Alessandria, mediante una ridefinizione delle aree di ricarica, focalizzata su questa porzione di territorio piemontese e una proposta di istituzione di aree di riserva delle acque sotterranee (RISE), da preservare ai fini idropotabili. Oltre alle classiche tecniche di monitoraggio idrodinamico e geochimico, le acque sono state campionate in una rete di monitoraggio dedicata, costituita da pozzi con filtri tra 80-300 metri di profondità, combinando le datazioni radiometriche $\left({ }^{14} \mathrm{C}\right.$ e $\delta^{13} \mathrm{C}$ del carbonio inorganico disciolto - DIC) con traccianti antropogenici $\left(\mathrm{CFC}, \mathrm{SF}_{6}\right)$, indicatori di ricarica recente o di fenomeni di miscelazione. La composizione degli isotopi stabili $\left(\delta^{2} \mathrm{H}\right.$ e $\delta^{18} \mathrm{O}-\mathrm{H}_{2} \mathrm{O}$ ) nelle precipitazioni nevose e pluviali è stata controllata durante un periodo di monitoraggio annuale, definendo una retta isotopica meteorica locale; è stata altresì verificata la variazione stagionale di contenuto in isotopi stabili nei principali corsi d'acqua e nei pozzi con filtri in acquifero profondo. Il modello numerico tridimensionale agli elementi finiti è stato implementato in ambiente FEflow, calibrato sulla base dei dati

Keywords: groundwater age, groundwater flow, isotopes, numerical modeling, protection.

Parole chiave: età delle acque sotterranee, flusso delle acque sotterranee, isotopi, modellazione numerica, protezione.

\section{Elena COGO 奉”?}

Nicola QUARANTA

GEO engineering s.r.l., Torino (TO), Italy

cogo@geoengineering.torino.it

\section{Adriano SIMONI}

EGATO6, Alessandria (AL), Italy

simoni@ato6alessandrino.it

Elisa SACCHI

Università di Pavia-DSTA (PV), Italy

elisa.sacchi@unipv.it

\section{Mariachiara CASCHETTO}

DISAA, Università degli Studi di Milano, via Celoria 2, 20133 (MI), Italy IT2E Isotope Tracer Technologies Europe srl (MI), Italy mariachiara.caschetto@it2europe.com

Massimo MARCHESI

IT2E Isotope Tracer Technologies Europe srl (MI), Italy massimo.marchesi@it2europe.com

Ricevuto/Received: 14 October 2019-Accettato/Accepted: 10 March 2020 Pubblicato online/Published online: 30 March 2020

This is an open access article under the CC BY-NC-ND license:

http://creativecommons.org/licenses/by-nc-nd/4.0/

(C) Associazione Acque Sotterranee 2020 di monitoraggio disponibili ed è stato utilizzato come strumento di supporto nella delimitazione delle aree di ricarica, a partire dall'analisi della distribuzione dei flussi. Tale modello si è reso fondamentale nella delimitazione delle aree di riserva (RISE), poiché in grado di simulare i deflussi sotterranei utilizzando sia condizioni di trasporto puramente advettivo (tecnica del particle tracking), sia condizioni più realistiche di trasporto advettivo $\mathrm{e}$ dispersivo, introducendo opportuni parametri di dispersività e utilizzando la distribuzione di Life Time Expectancy. Lutilizzo integrato delle tradizionali tecniche di analisi del sistema di flusso idrogeologico regionale basate sul monitoraggio idrochimico e degli isotopi stabili, unitamente alle tecniche di datazione basate sugli isotopi naturali radioattivi e antropogenici, ha fornito un valido supporto nella validazione del modello numerico di flusso e trasporto, finalizzato a definire le aree di ricarica e le zone di riserva dedicate alla futura estrazione in acquiferi profondi a scopo idropotabile

Abstract: The aim of the study was to set up a protection system from pollution of the deep aquifer of the Alessandria basin, by redefining the recharge areas, focused on this portion of Piedmont territory, and therefore by creating some reserve areas of deep groundwater, to be preserved for future buman drinking purposes. In addition to the classical bydrodynamic and geochemical monitoring techniques, the groundwaters were analyzed with reference to a monitoring network of 25 selected wells with deep screens (80-300 $\mathrm{m}$ below ground surface) combining radiochemical dating $\left({ }^{14} \mathrm{C}\right.$ and $\delta^{13} \mathrm{C}$ of dissolved inorganic carbon - DIC) with anthropogenic tracers $\left(C F C s, S F_{6}\right)$ as indicators of recent rechargel mixing. Stable isotope composition $\left(\delta^{2} \mathrm{H}\right.$ and $\left.\delta^{18} \mathrm{O}-\mathrm{H}_{2} \mathrm{O}\right)$ was assessed during a 1-year sampling of snow-rain precipitations gauges distributed in altitude in order to define Local Meteoric Water Lines; the isotopic composition was also seasonally measured for the main rivers and in selected deep-wells. The 3D numerical model was implemented in FEflow platform and calibrated on the basis of the available monitoring data; it was used as a support tool in the delimitation of the recharge areas, starting from the analysis of the distribution of flows. This model was crucial for delimiting the "reserve areas", since it was able to simulate groundwater flows using both purely advective transport conditions (particle tracking technique), and more realistic conditions of advective and dispersive transport, by introducing dispersive parameters and using the Life Time Expectancy (LTE) reservoir distribution. The integrated use of "traditional" techniques of regional groundwater flow system monitoring (bydrochemistry, stable isotopic composition) and of dating techniques based on radioactive isotopes and anthropogenic tracers, provided a reliable support to the validation of flow and transport simulation model, oriented to identify recharge areas and "reserve areas" of future extraction of deep groundwater for drinking purpose. 


\section{Introduzione}

Il presente contributo si riferisce ad un bacino idrogeologico di circa $1000 \mathrm{Km}^{2}$ in provincia di Alessandria, (Piemonte orientale, IT), in cui l'Ente di Governo dell'Ambito Territoriale Ottimale $n^{\circ} 6$ (EGATO6) svolge le funzioni di governance e programmazione del Servizio Idrico Integrato.

Obiettivo del lavoro è caratterizzare qualitativamente e quantitativamente il sistema di flusso degli acquiferi profondi utilizzati a scopo idropotabile, nell'intervallo di profondità tra 80-300 m da p.c. Lo studio, condotto combinando tecniche tradizionali (stratigrafiche, idrogeologiche ed idrochimiche) con approcci specialistici (isotopi stabili ed instabili, traccianti antropogenici), è finalizzato ad una più approfondita definizione delle aree di ricarica, già delimitate a scala regionale, e delle zone di riserva (RISE), da proporre nella pianificazione come porzioni di acquifero profondo da preservare per futuri utilizzi di pregio, considerata l'elevata disponibilità quantitativa e le favorevoli caratteristiche geochimiche.

\section{Materiali e metodi \\ Metodologia generale e flusso delle attività}

La prima fase di lavoro si è concentrata sulla sistematizzazione delle conoscenze stratigrafiche e idrogeologiche di base, attraverso una rigorosa selezione delle basi-dati di settore esistenti.

Questo ha permesso di fissare le condizioni idrologicoidrauliche al contorno e interne al dominio di studio. Sono state eseguite nel 2018 due campagne stagionali di monitoraggio piezometrico, idrochimico e isotopico in una trentina di pozzi profondi, misurate 7 sezioni fluviali in fase di ricarica tardoinvernale e di esaurimento estiva ed è stata attivata una rete di pluviometri situati a varie quote e posizioni in area appenninica adiacente al bacino, con strumenti idonei al campionamento delle precipitazioni per analisi isotopica, con la collaborazione del Laboratorio di Idrochimica del Dipartimento di Scienze della Terra dell'Università di Torino e del laboratorio ISO4 (Torino). In un sottoinsieme di pozzi rappresentativi sono stati

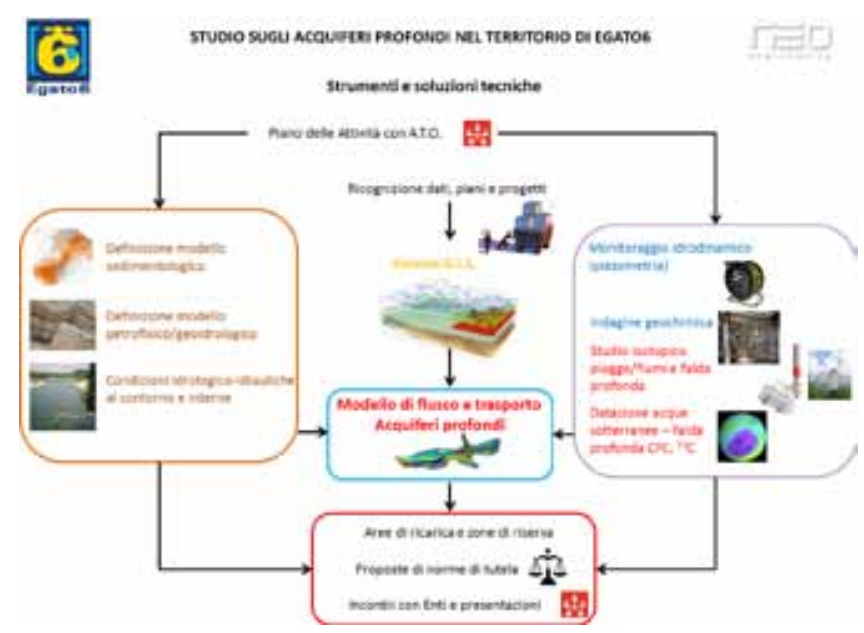

Fig. 1 - Flusso delle attività Fig. 1 - Workflow. misurati i traccianti naturali e antropogenici, per ottenere prime indicazioni sull'età delle acque circolanti in acquiferi profondi, verificare fenomeni di miscelamento e il grado di confinamento. I dati sperimentali sono stati confrontati con un modello matematico di flusso e trasporto agli elementi finiti, per completare il quadro degli strumenti di analisi quantitativa e supportare le opzioni di tutela della risorsa con la maggiore gamma di strumenti.

Infine, i risultati ottenuti sono stati condivisi ed analizzati criticamente in diverse sessioni di confronto con i portatori di interesse (Enti territoriali e di ricerca, professionisti).

Il flusso delle attività è rappresentato in Figura 1.

\section{Modello idrogeologico e sedimentologico}

Lo studio idrogeologico del sistema acquifero profondo rappresenta un affinamento del modello sedimentologico, petrofisico e geoidrologico regionale definito in Irace et al. (2009).

Il bacino alessandrino costituisce una struttura deposizionale ad andamento centripeto asimmetrico, soggetta ad una deformazione sinclinale-sinforme, il cui asse è riconosciuto nella Carta Geologica d'Italia (scala 1:100.000, Foglio 70 - Alessandria) in direzione WNW-SSE tra il F. Tanaro e la confluenza Bormida-Orba, dove si verifica il massimo approfondimento depocentrale dei depositi terziari (Braga G. et alii 1976). L'interpretazione integrata dei profili sismici e dei pozzi esplorativi AGIP di Quargnento e Novi Ligure pone in evidenza lo sviluppo dell'assetto bacinale sulle strutture deformative condizionanti la successione deposizionale terziaria, determinando una marcata depressione della superficie Pliocenica basale (Fig. 2).

Tale modello esplicita la relazione tra i sintemi deposizionali aventi significato crono-stratigrafico e i gruppi acquiferi (e le relative unità idrogeologiche di gruppo), in accordo allo schema riportato nella figura 2. Lo studio si è focalizzato soprattutto sui gruppi acquiferi B e C (Sintemi Q1 e P3, compresi tra la successione del Pliocene medio e del Pleistocene medio; Fig. 2), maggiormente utilizzati a scopo idropotabile per le favorevoli caratteristiche quali-quantitative.

Per l'affinamento del modello regionale sono stati utilizzati nuovi dati desunti da oltre 260 stratigrafie di pozzi, filtranti esclusivamente l'acquifero profondo ed aventi profondità variabili fra 31 e $1716 \mathrm{~m}$ da piano campagna, reperite presso la Provincia di Alessandria e i gestori di infrastrutture idriche dell'EGATO.

Le colonne stratigrafiche utilizzate (Fig. 3) mostrano una diminuzione di numero, sino alla progressiva scomparsa, di quelle riferite a pozzi profondi nella zona di platea sepolta dei depositi terziari “Tortona-Montecastello", definita dai sistemi di thrust. Ad eccezione del concentrico di Alessandria, l'area occidentale tra i F. Tanaro e il T. Belbo non risulta di norma ricca di pozzi profondi.

Per ricostruire la piezometria, sono stati scelti 39 pozzi, con profondità del primo filtro sotto gli $80 \mathrm{~m}$ dal piano campagna, filtranti esclusivamente i gruppi acquiferi di riferimento del presente studio (B, C secondo Irace et al. 2009). 


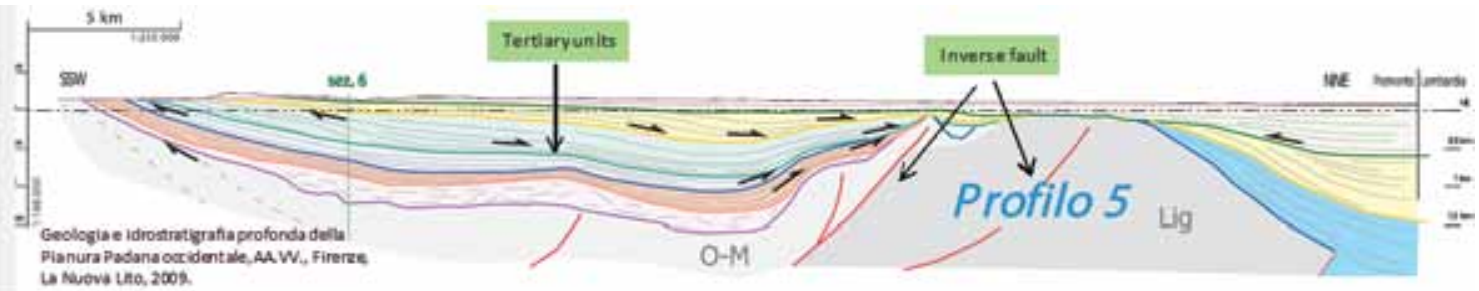

\begin{tabular}{|c|c|c|c|c|c|c|}
\hline$=$ & & 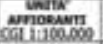 & unerwe savount & momen & 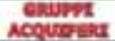 & 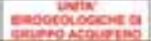 \\
\hline$\infty$ & 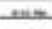 & & & & & \\
\hline & - & incosicin & & (1023. & A & At Al A \\
\hline & $=$ & & 2Z2Ees: & Q. & B & \\
\hline & $=$ & & & & & \\
\hline i & & 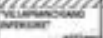 & & $P$ Ps & c & cr en enil \\
\hline$\stackrel{0}{c}$ & $\ldots$ & & 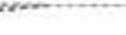 & $=10$ & D & \\
\hline E & - & $=$ & & I & 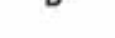 & and \\
\hline & & & 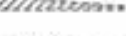 & 1 & $E$ & $t i$ \\
\hline$\infty$ & 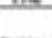 & we & & 642 & $\mathbf{F}$ & rii rai \\
\hline$=0$ & $=$ & 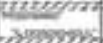 & & 1082 & G & \\
\hline
\end{tabular}

Fig. 2 - Sezione stratigrafica trasversale e schema dei rapporti tra unità sedimentarie e gruppi acquiferi (Fonte: Irace et al. 2009, riproduzione autorizzata dagli Autori, comunicazione personale 10.10.19).

Fig. 2 - Geological cross-section and scheme of the relationships between sedimentary units and aquifer groups (Source: Irace et al. 2009, reproduction allowed by the Authors, personal communication 10.10.19).

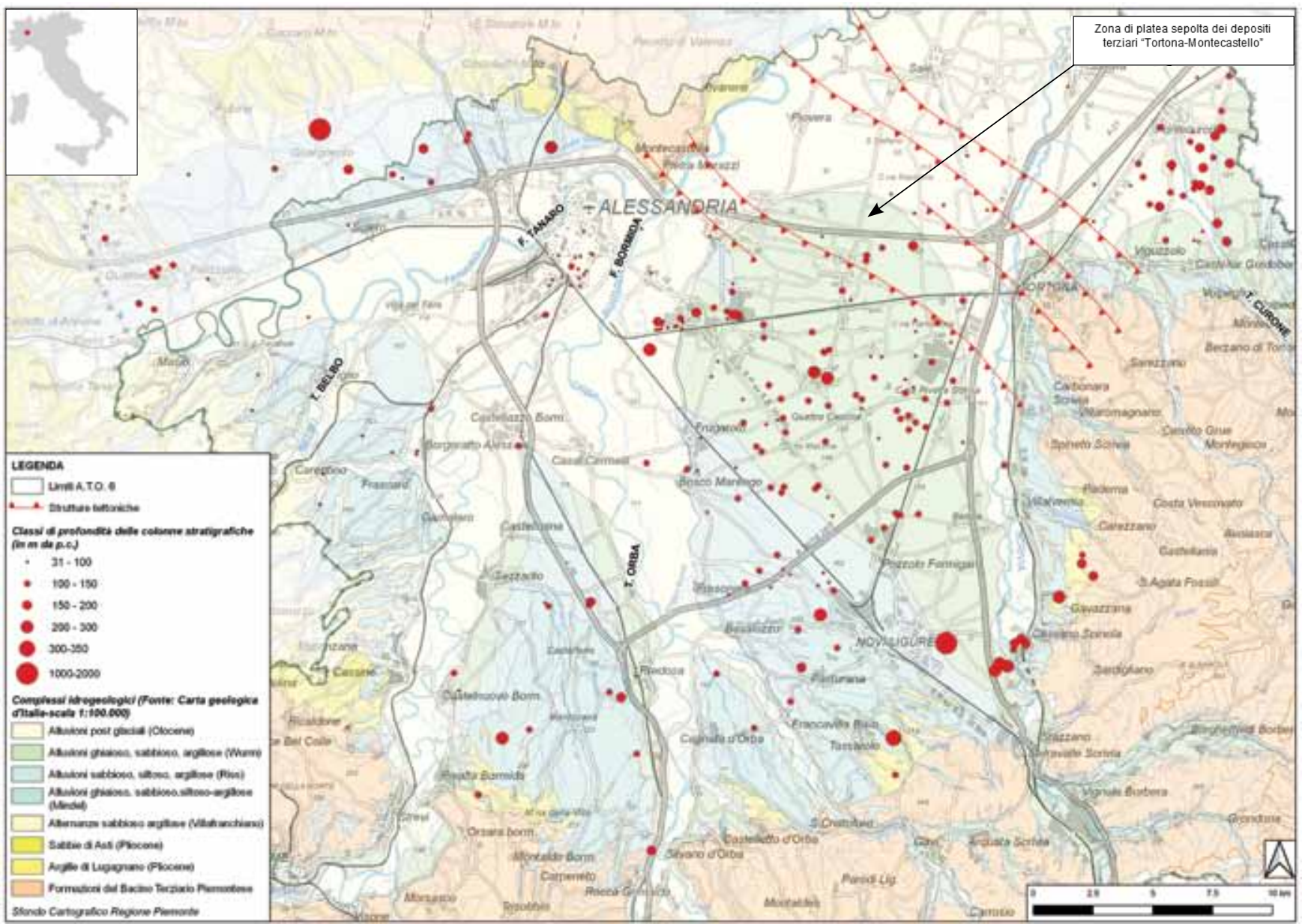

Fig. 3 - Classi di profondità delle colonne stratigrafiche analizzate.

Fig. 3 - Depth classes of the analyzed stratigraphic columns. 


\section{Monitoraggio idrogeochimico}

Nelle due campagne di monitoraggio annuale sono state eseguite 53 misure dei parametri chimico-fisici, in sito con sonda multiparametrica e tramite analisi in laboratorio su anioni-cationi di base e metalli ( $\mathrm{T}, \mathrm{pH}$, cond. el. spec., DO, pot. Redox, $\mathrm{Li}, \mathrm{Na}, \mathrm{K}, \mathrm{NH}_{4}, \mathrm{Ca}, \mathrm{Mg}, \mathrm{F}, \mathrm{Cl}, \mathrm{Br}, \mathrm{PO}_{4}, \mathrm{HCO}_{3}$, $\mathrm{CO}_{3}, \mathrm{SO}_{4}, \mathrm{NO}_{2}, \mathrm{NO}_{3}, \mathrm{Ni}, \mathrm{Fe}, \mathrm{Mn}, \mathrm{Cr}_{\mathrm{VI}}$, TOC). La facies idrochimica più diffusamente riscontrata corrisponde, secondo le rappresentazioni dei diagrammi di Schoeller (1962) e Stiff (1951), alla facies bicarbonato-calcico-magnesiaca (Fig. 4)

La mineralizzazione delle acque di circolazione profonda risulta maggiore nei pozzi ai margini meridionale ed orientale del bacino (Fig. 5), alimentati da acque del Conglomerato di Cassano Spinola. I pozzi nella zona centrale del bacino sono riferibili ad acque dei depositi continentali e di transizione in facies "Villafranchiana" e, in subordine, delle "Sabbie di Asti". La minore mineralizzazione nella zona centrale del bacino potrebbe essere giustificata da una miscelazione con acque di falde superficiali, più recenti e meno evolute. Alternativamente, potrebbe trattarsi di acque geneticamente riconducibili ad apporti alpini durante le fasi glaciali/ interglaciali, contraddistinti da basse mineralizzazioni. Tale ipotesi, che non è strettamente esclusiva/alternativa rispetto ai fenomeni di miscelamento con acque più superficiali, parrebbe confermata anche dalla datazione radiometrica delle acque prelevate nei pozzi di monitoraggio in questo settore.

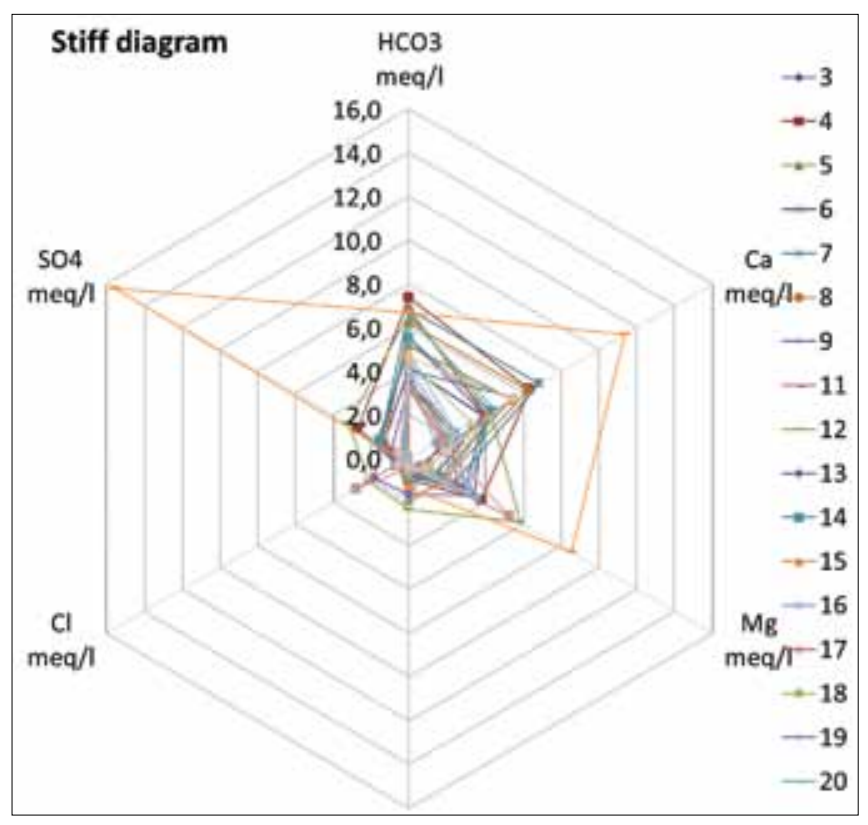

Fig. 4 - Diagramma di facies idrochimica di Stiff (1951).

Fig. 4 - Stiff diagram (1951) with hydrochemical facies.

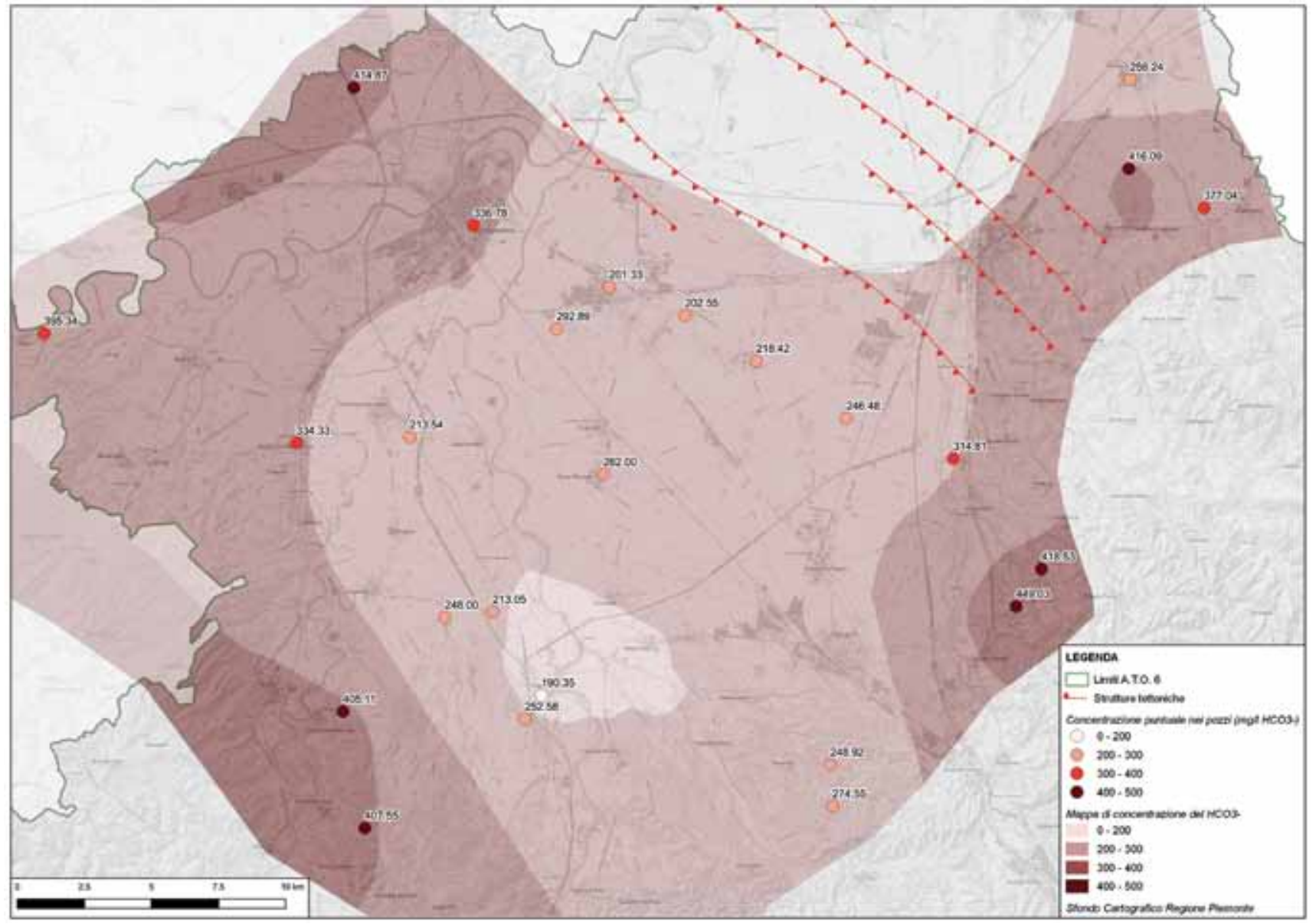

Fig. 5 - Distribuzione della concentrazione di ione bicarbonato.

Fig. 5 - Areal distribution of bicarbonate ion. 


\section{Monitoraggio isotopico}

E' stata definita sperimentalmente la retta meteorica locale di frazionamento isotopico delle precipitazioni con l'altitudine, avvalendosi di tre stazioni meteopluviometriche rappresentate in figura 6 , disposte tra la sommità appenninica del Monte
Beigua ed Acqui Terme, ai limiti della zona di ricarica meridionale del bacino. Gli apporti pluviali sono riferiti alla provenienza delle masse d'aria umida, prevalentemente di impronta atlantica e solo in subordine di origine più fredda, artica o dall'Europa Orientale.

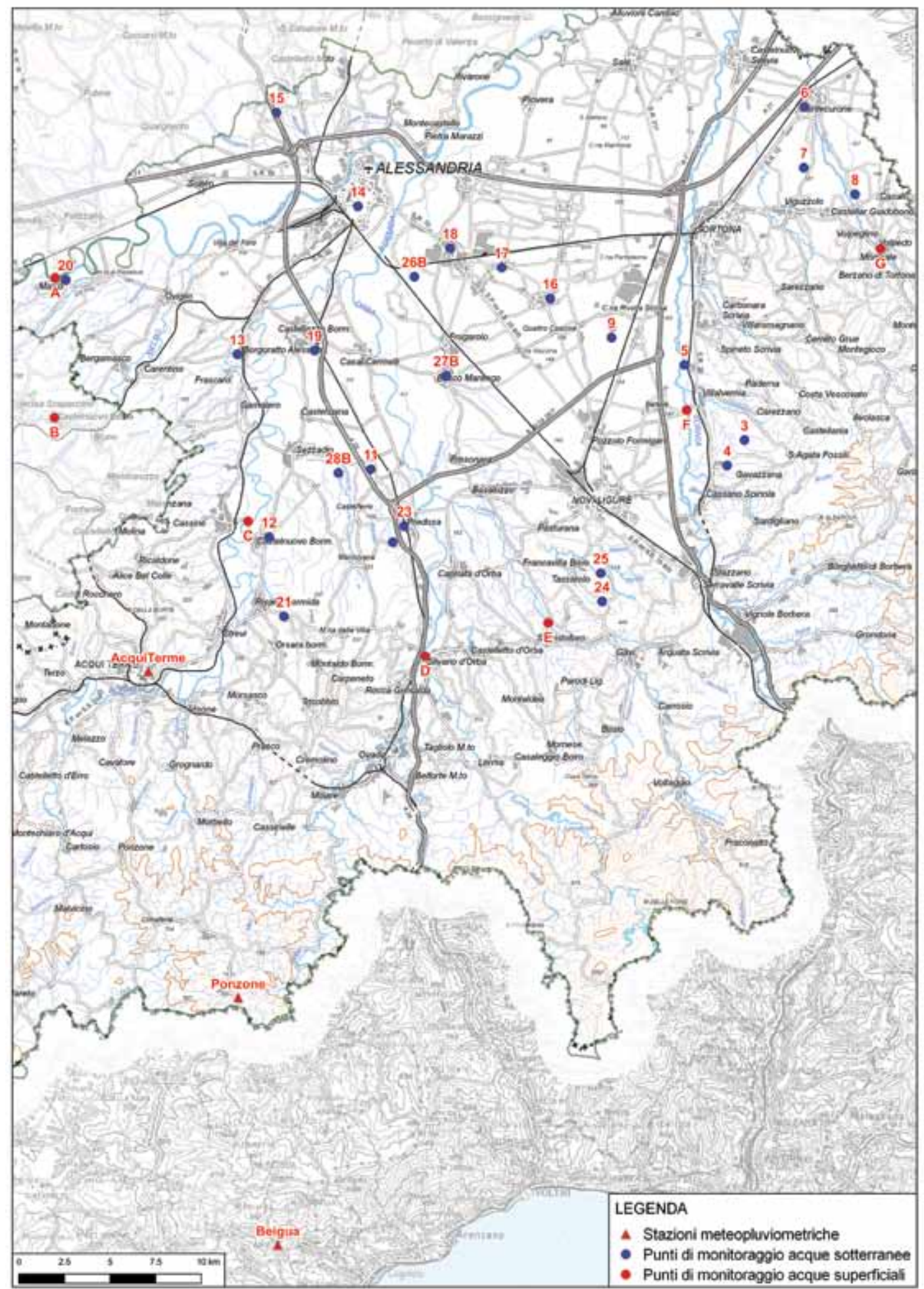

Fig. 6 - Stazioni meteopluviometriche utilizzate per la ricostruzione della retta meteorica locale, punti di monitoraggio delle acque sotterranee e superficiali. Fig. 6 - Meteopluviometric stations used to design a Local Meteoric Water Line, groundwater and streams monitoring points. 
Si osservano correlazioni ad elevato coefficiente di regressione tra composizione isotopica delle precipitazioni e temperatura media mensile.

La composizione isotopica media ponderata delle precipitazioni nella zona di ricarica ai margini del sistema idrogeologico di pianura è

$\delta^{18} \mathrm{O}=-8.4\left(1000 * \delta^{18} \mathrm{O}\right.$ vs $\left.\mathrm{V}-\mathrm{SMOW} 2\right)$

$\delta^{2} \mathrm{H}=-54.3\left(1000 * \delta^{2} \mathrm{H}\right.$ vs $\left.\mathrm{V}-\mathrm{SMOW} 2\right)$

$$
\delta=\left[\left(\frac{R_{\text {campione }}}{R_{\text {standard }}}\right)-1\right] \cdot 10^{3}
$$

Il frazionamento isotopico delle precipitazioni con la quota (h in m.l.m.) è espresso dalle seguenti correlazioni locali (per brevità nel seguito i dati di composizione isotopica verranno espressi in "notazione delta"), ottenute tramite media ponderale delle pendenze e dell'intercetta sull'asse delle ordinate, riferita alle altezze di precipitazione mensile (Fig. 7).

$\delta^{2} \mathrm{H} \%=-0.017 \mathrm{~h}-50,61 \%$

$\delta^{18} \mathrm{O} \%=-0.002 \mathrm{~h}-7,72 \%$

La correlazione per il deuterio non è dissimile da quella ottenuta per il vicino bacino delle Terme di Acqui (Marini et al. 2000)

$$
\delta^{2} \mathrm{H} \%=-0.01507 \mathrm{~h}-49,57 \%
$$

Il campionamento delle acque superficiali si riferisce a fasi idrologiche di morbida primaverile, magra estiva e morbida autunnale.

La composizione isotopica media dei corsi d'acqua campionati risulta:

$$
\begin{aligned}
& \delta^{18} \mathrm{O} \%=-9.0 \pm 1.5(\mathrm{~m} \pm 1 \sigma) \\
& \delta^{2} \mathrm{H} \%=-59.2 \pm 12.8(\mathrm{~m} \pm 1 \sigma)
\end{aligned}
$$

$\mathrm{Si}$ osserva un arricchimento isotopico tra la morbida primaverile e la magra estiva, nell'ordine di grandezza del $12 \%$ per il $\delta^{18} \mathrm{O}$ e del $15 \%$ per il $\delta^{2} \mathrm{H}$ (Tab. 1 , Tab. 2); tale arricchimento in isotopi pesanti è differenziato nei diversi bacini afferenti al dominio di studio: massimo ( $\geq 30 \%)$ nel bacino del T. Belbo; con valori $<10 \%$ nel bacino del $\mathrm{F}$. Tanaro, minimo $(<7 \%)$ nei bacini del T. Scrivia e T. Curone.
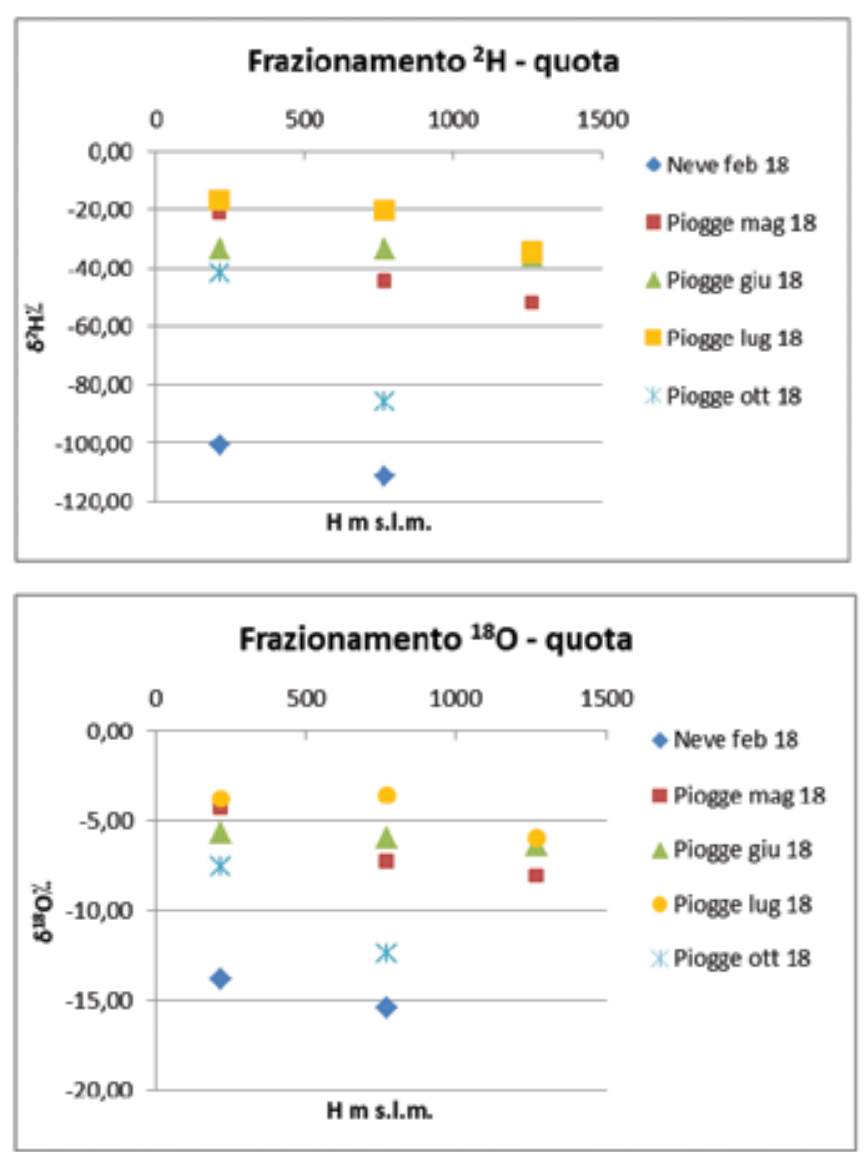

Fig. 7 - Diagrammi di frazionamento isotopico delle precipitazioni.

Fig. 7 - Diagrams showing isotopic fractioning with elevation.

Nei pozzi campionati nelle due distinte fasi di ricarica stagionale e di esaurimento, il $90 \%$ presenta una variazione del $\delta^{18} \mathrm{O}<5 \%$, indicativa di un confinamento medio-alto dell'acquifero e di bassa vulnerabilità. Solo il $10 \%$ dei pozzi mostra variazioni del $\delta^{18} \mathrm{O}$ compresa tra $5 \%$ - 10\%, indicativa di condizioni semi-confinate e di una vulnerabilità mediobassa. Alcuni pozzi, non distanti dalle aree di ricarica, presentano maggiori variazioni isotopiche stagionali, indicative di una interconnessione con la falda superficiale in

\begin{tabular}{|c|c|c|c|c|c|c|}
\hline Mese misure 2018 & $\begin{array}{l}\text { Termine "a" } \\
\text { della retta di } \\
\text { frazionamento } \\
\text { (pendenza) } \\
\text { Valore mensile }\end{array}$ & $\begin{array}{l}\text { Termine "b" } \\
\text { della retta di } \\
\text { frazionamento } \\
\text { (intercetta asse } \\
\text { ordinate) } \\
\text { Valore mensile }\end{array}$ & $\begin{array}{l}\text { Altezza di } \\
\text { precipitazione } \\
\text { media mensile } \\
(\mathrm{mm})\end{array}$ & $\begin{array}{l}\text { Peso relativo della } \\
\text { precipitazione } \\
\text { mensile nel } \\
\text { periodo }\end{array}$ & $\begin{array}{l}\text { Termine "a" } \\
\text { della retta di } \\
\text { frazionamento } \\
\text { (pendenza) } \\
\text { Valore ponderato }\end{array}$ & $\begin{array}{l}\text { Termine "b" } \\
\text { della retta di } \\
\text { frazionamento } \\
\text { (intercetta asse } \\
\text { ordinate) } \\
\text { Valore ponderato }\end{array}$ \\
\hline feb & $-0,0191$ & $-96,497$ & 118,00 & $38 \%$ & $-0,00718$ & $-36,286$ \\
\hline mag & $-0,0296$ & $-17,095$ & 81,10 & $26 \%$ & $-0,00765$ & $-4,418$ \\
\hline giu & $-0,0022$ & $-32,623$ & 84,70 & $27 \%$ & $-0,00059$ & $-8,806$ \\
\hline \multirow[t]{2}{*}{ lug } & $-0,0168$ & $-11,479$ & 30,00 & $10 \%$ & $-0,00161$ & $-1,097$ \\
\hline & & & 313,80 & $100 \%$ & $-0,017$ & $-50,61$ \\
\hline
\end{tabular}
contatto idraulico con le acque meteoriche e fluviali.

Tab. 1 - Dati utilizzati per la definizione della retta di frazionamento isotopico altitudinale del ${ }^{2} \mathrm{H}$.

Tab. 1 - Data used for the definition of the altitudinal isotopic fractionation line of ${ }^{2} \mathrm{H}$. 
Tab. 2 - Dati utilizzati per la definizione della retta di frazionamento isotopico altitudinale del ${ }^{18} \mathrm{O}$.

Tab. 2 - Data used for the definition of the altitudinal isotopic fractionation line of ${ }^{18} \mathrm{O}$.

\begin{tabular}{|l|l|l|l|l|l|l|}
\hline & $\begin{array}{l}\text { Termine “a” } \\
\text { della retta di } \\
\text { frazionamento } \\
\text { (pendenza) } \\
\text { Valore mensile }\end{array}$ & $\begin{array}{l}\text { Termine "b" } \\
\text { della retta di } \\
\text { frazionamento } \\
\text { (intercetta asse } \\
\text { ordinate) } \\
\text { Valore mensile }\end{array}$ & $\begin{array}{l}\text { Altezza di } \\
\text { precipitazione } \\
\text { media mensile } \\
(\mathrm{mm})\end{array}$ & $\begin{array}{l}\text { Peso relativo della } \\
\text { precipitazione } \\
\text { mensile nel } \\
\text { periodo }\end{array}$ & $\begin{array}{l}\text { Termine "a" } \\
\text { della retta di } \\
\text { frazionamento } \\
\text { (pendenza) } \\
\text { Valore ponderato }\end{array}$ & $\begin{array}{l}\text { Termine "b" } \\
\text { della retta di } \\
\text { frazionamento } \\
\text { (intercetta asse } \\
\text { ordinate) } \\
\text { Valore ponderato }\end{array}$ \\
\hline feb & $-0,0029$ & $-13,188$ & 118,00 & $38 \%$ & $-0,00109$ & $-4,959$ \\
\hline mag & $-0,0036$ & $-3,8376$ & 81,10 & $26 \%$ & $-0,00093$ & $-0,992$ \\
\hline giu & $-0,0006$ & $-5,53$ & 84,70 & $27 \%$ & $-0,00016$ & $-1,493$ \\
\hline lug & $-0,0021$ & $-2,8714$ & 30,00 & $10 \%$ & $-0,0002$ & $-0,275$ \\
\hline & & & 313,80 & $100 \%$ & $-0,002$ & $-7,72$ \\
\hline
\end{tabular}

La composizione isotopica media dei pozzi in falda in pressione risulta:

$$
\delta^{18} \mathrm{O} \%=-8.8 \pm 0.52 \%(\mathrm{~m} \pm 1 \sigma)
$$

$\delta^{2} \mathrm{H} \%=-57.6 \pm 3.75 \%(\mathrm{~m} \pm 1 \sigma)$

La quota media di ricarica "isotopica" degli acquiferi profondi, calcolata confrontando la composizione isotopica media dei pozzi con il frazionamento isotopico altitudinale (quota- $\delta^{2} \mathrm{H}$ e quota- $\delta^{18} \mathrm{O}$ ) è compresa tra $210-270 \mathrm{~m}$ s.l.m.

Nella figura 9 è stata rappresentata la distribuzione areale della composizione isotopica media delle acque sotterranee e superficiali, e, dall'analisi della stessa, è possibile riconoscere "distretti ad impronta isotopica" connotati da un progressivo impoverimento isotopico procedendo dal margine appenninico verso il F. Tanaro, dovuto alla diversa impronta isotopica dei rispettivi bacini di alimentazione. Il settore prossimo al F. Tanaro (Nord-Ovest del bacino) si connota per i valori inferiori, i corsi d'acqua nel settore sud-orientale (F. Scrivia, T. Lemme, T. Orba) si connotano per i valori superiori, nella zona centrale i valori nei pozzi riflettono condizioni di miscelazione variabile; il distretto delle acque profonde lungo il Bormida risulta ben differenziato, intorno a valori intermedi tra i due precedenti.

\section{Datazione delle acque profonde}

Si è adottato un approccio "multi-tracciante" per la stima delle "età" delle acque profonde, considerando il campione prelevato in pozzo come una miscela di acque di differente origine, residenti nel sottosuolo per diversi intervalli temporali. Le analisi sono state condotte dal laboratorio IT2E Isotope Tracer Technologies Europe srl e da Spurenstofflabor (DE).

Si sono adottate due diverse tecniche complementari tra loro, per un totale di 10 campioni, 5 dei quali di acque meno mineralizzate e più prossime a zone di ricarica, misurando la concentrazione dei gas disciolti CFC e $\mathrm{SF}_{6}$ (traccianti antropogenici immessi nell'ambiente a partire dal 1940; IAEA 2006), e 5 di acque provenienti da pozzi in falde confinate profonde, con misura del radiocarbonio o C-14 (corretta sulla base del valore di $\delta^{13} \mathrm{C}$ ). I risultati confermano la prossimità delle acque meno mineralizzate alle zone di ricarica (età tra 26 e 67 anni), e, al contrario, la presenza di circuiti di flusso

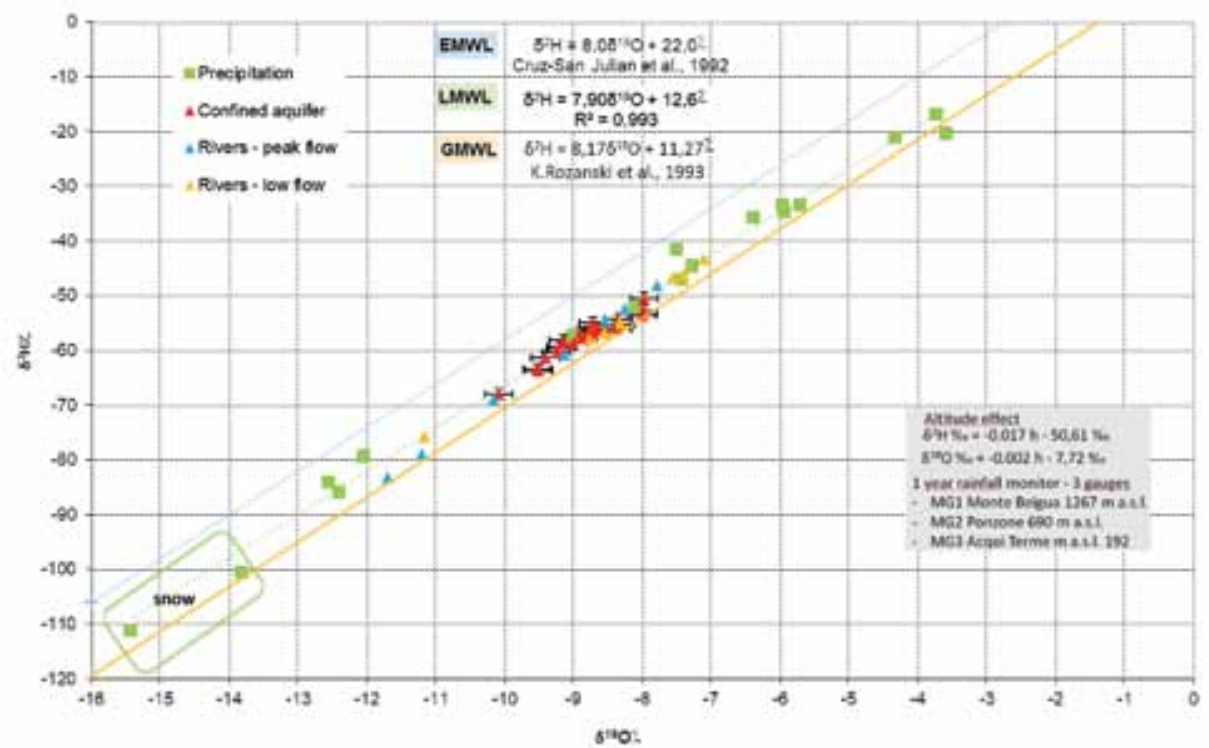

Fig. 8 - Sintesi dei dati isotopici, confronto tra retta meteorica locale LMWL e rette globali (EMWL, GMWL).

Fig. 8 - Isotopic data synthesis, comparison between local meteoric line LMWL and global. 


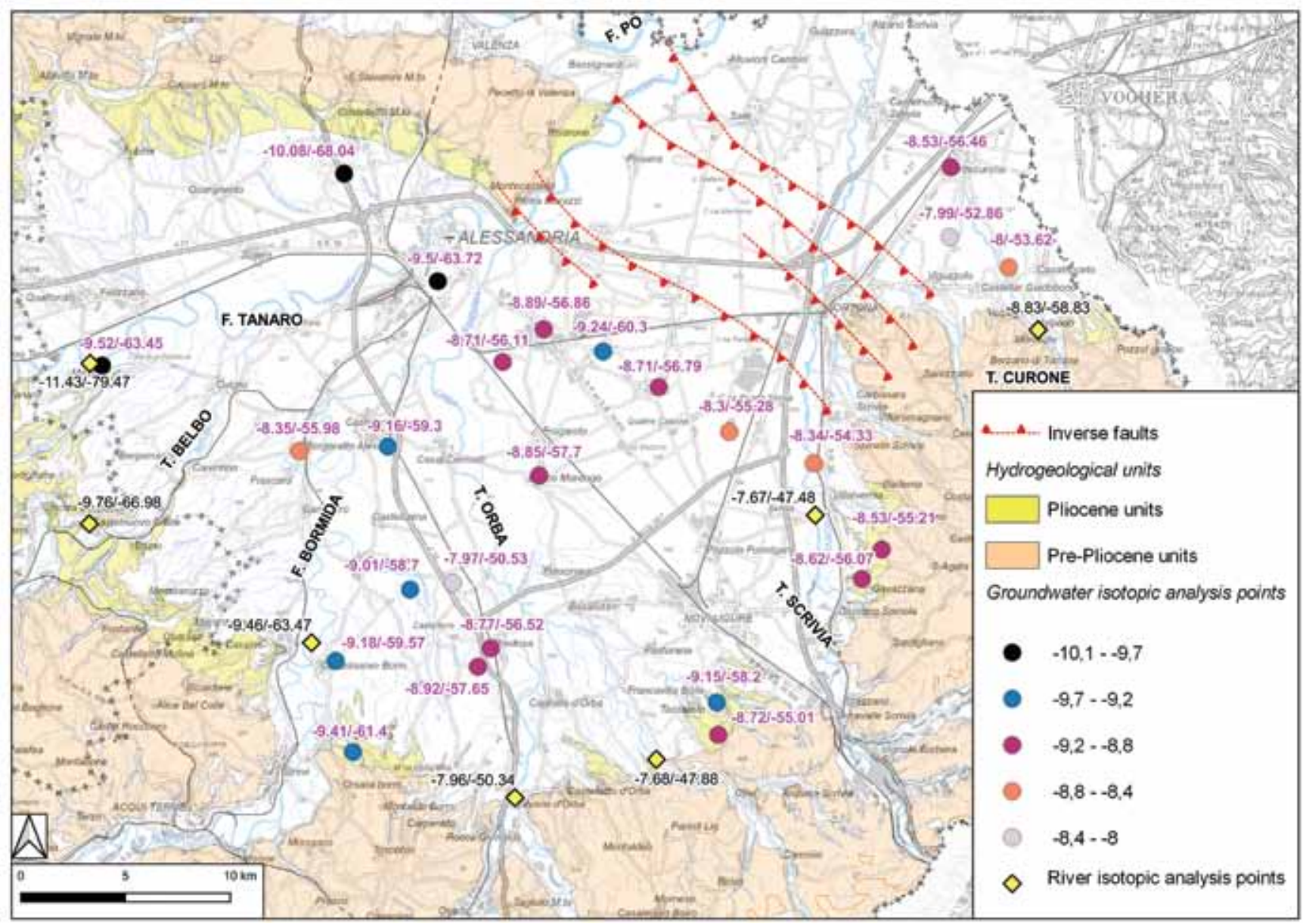

Fig. 9 - Distribuzione dei dati isotopici nei pozzi e corsi d'acqua $\left(\delta^{18} \mathrm{O} / \delta^{2} \mathrm{H}\right)$.

Fig. 9 - Isotopic values in wells and rivers $\left(\delta^{18} \mathrm{O} / \delta^{2} H\right)$.

lunghi e profondi intercettati da alcuni pozzi in pressione, in grado di richiamare acque "tardo-Pleistoceniche" (età da 2.400 a 18.900 anni dal presente). I due metodi analitici sono complementari, in quanto la misura dei $\mathrm{CFC}_{-} \mathrm{SF}_{6}$ è appropriata per le acque dell'ultimo settantennio, mentre la misura con radio-carbonio è orientata alla stima dell'età di acque antecedenti al $1950(\mathrm{BP}=0)$.

Si nota una buona correlazione tra età radiometrica calcolata con il metodo del radiocarbonio e profondità media dei filtri nei pozzi (Fig. 10).

Le discordanze di età relative ad un pozzo profondo nella zona Sud-Est del bacino potrebbero essere interpretate in relazione alla presenza di impulsi di ricarica recenti veicolati lungo lineazioni strutturali (fratture), in grado di raggiungere il serbatoio più profondo, prevalentemente alimentato con acque più antiche.

\section{Implementazione del modello di calcolo}

E' stato applicato al caso in esame il software FEFLOW (Finite Element subsurface FLOW system), uno tra i più sofisticati codici numerici disponibili per la simulazione dei processi di flusso e trasporto in mezzi porosi, in condizioni sature ed insature, interfacciato con gli strumenti di cartografia GIS, sia in fase di pre-processing che in fase di analisi dei risultati.

Nel piano orizzontale i limiti del dominio di calcolo sono controllati dai lineamenti geomorfologici e dai limiti idrogeologici del bacino (Fig. 11). Verticalmente il modello geologico è suddiviso in 5 unità idrogeologiche, conformi all'assetto idrostratigrafico precedentemente accertato (Tab. 3).

La griglia di calcolo è rifinita sia orizzontalmente che verticalmente, suddividendo i gruppi acquiferi in strati di calcolo a minore spessore, per affinare la risoluzione computazionale (Fig. 12).

Le dimensioni sui due assi orizzontali del modello regionale sono di $34.6 * 43.5 \mathrm{~km}$, il dislivello sull'asse verticale è $453 \mathrm{~m}$, la griglia di calcolo comprende 61.164 celle triangolari e 35.420 nodi.

I parametri idrodinamici sono distribuiti nei singoli strati di calcolo tenendo conto della variabilità verticale e spaziale dei gruppi acquiferi, a partire dall'analisi delle Banche Dati, nonché sulla base della bibliografia di settore. Per i gruppi acquiferi filtrati dalla maggior parte dei pozzi a stratigrafia nota, la conducibilità idraulica è correlata ai dati di portata specifica, e i dati sono stati interpolati arealmente (Fig. 14). 

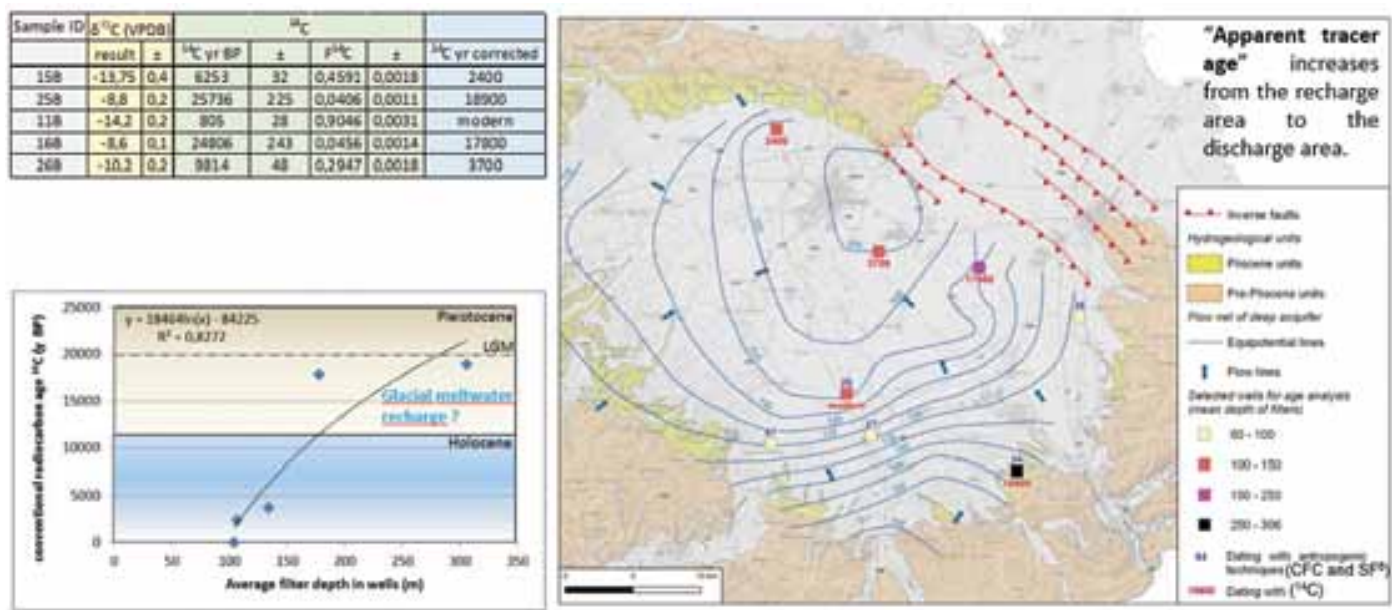

Fig. 10 - Distribuzione dei dati di età radiometrica ed apparente, da traccianti antropogenici.

Fig. 10 - Radiometric age distribution and apparent age, from anthropogenic tracers.

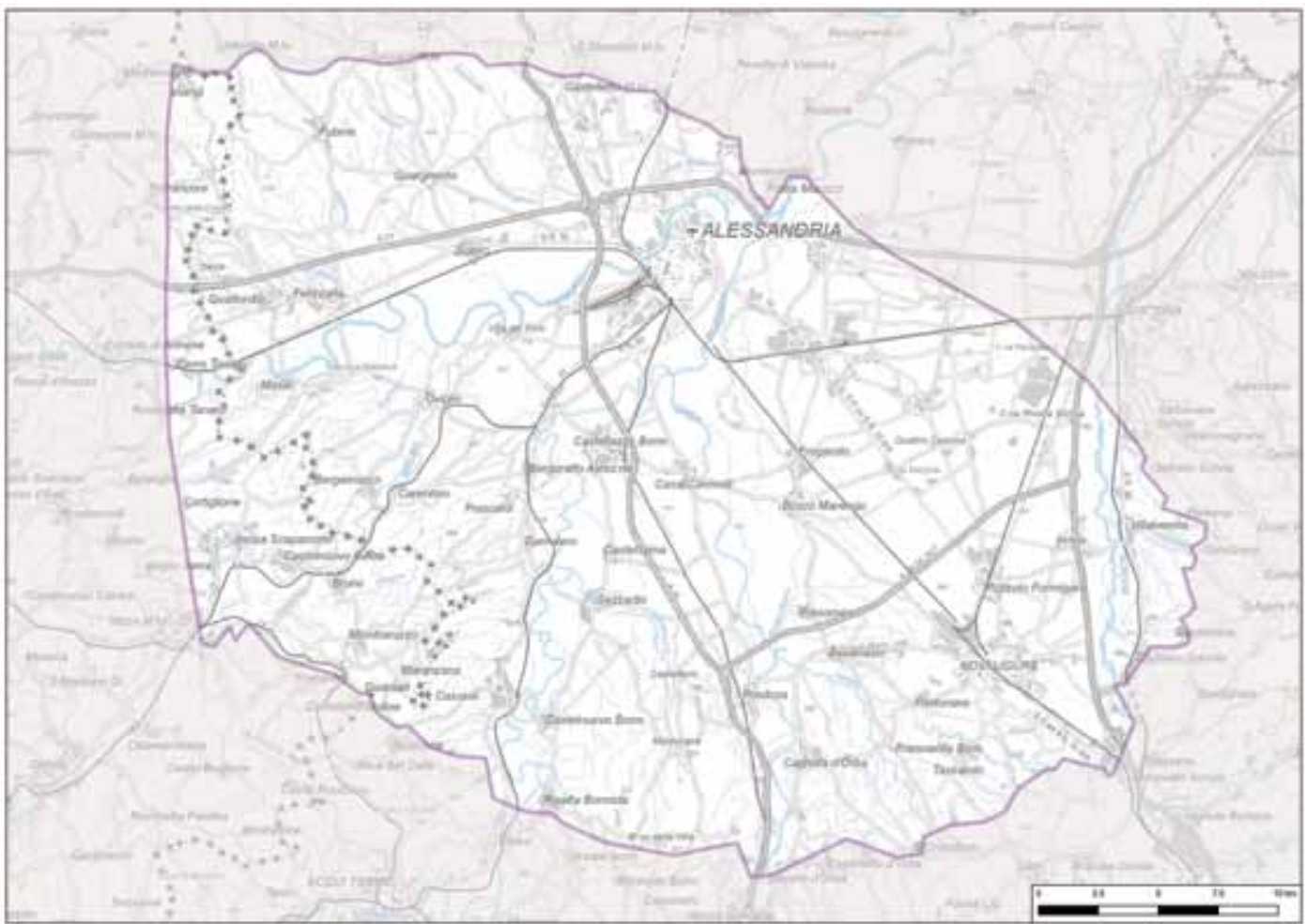

Fig. 11 - Poligono di delimitazione del dominio del modello.

Fig. 11 - Polygonal delimitation of model domain.

Tab. 3 - Discretizzazione verticale del modello e parametri idrodinamici.

Tab. 3 -Vertical discretization of the model and assignment of hydrodynamic parameters.

\begin{tabular}{|c|c|c|c|}
\hline Layer & Gruppi acquiferi & $\mathrm{Kx}, \mathrm{Ky}(\mathrm{m} / \mathrm{s})$ & $\mathrm{Kz}(\mathrm{m} / \mathrm{s})$ \\
\hline 1 & UIG AI - UIG AII - UIG A IV & $1 * 10-4-1 * 10^{-5}$ & $1 * 10-05-1 * 10^{-6}$ \\
\hline $2 / 3 / 4$ & $\frac{\text { Unit B }}{\text { UIG BII }}$ & \multicolumn{2}{|c|}{$\begin{array}{l}\text { Interpolated distribution } \\
\text { (range } 3.3 * 10^{-5}-4.0 * 10^{-3} \text { ) }\end{array}$} \\
\hline $5 / 6$ & $\frac{\text { Unit C }}{\text { UIG CI - UIG CII }}$ & \multicolumn{2}{|c|}{$\begin{array}{l}\text { Interpolated distribution } \\
\text { (range } 3.3 * 10^{-5}-4.0 * 10^{-3} \text { ) }\end{array}$} \\
\hline $7 / 8$ & $\frac{\underline{\text { Unit D }}}{\text { UIG DI - UIG DIII - UIG DIV }}$ & $5.0 * 10^{-5}-1.0 * 10^{-7}$ & $5.0 * 10^{-6}-1.0 * 10^{-8}$ \\
\hline 9 & $\frac{\text { Unit E }}{\text { UIG EIII - UIG EIV }}$ & $5.0 * 10^{-5}-1.0 * 10^{-7}$ & $5.0 * 10^{-6}-1.0 * 10^{-8}$ \\
\hline
\end{tabular}


Nella definizione delle condizioni al contorno e all'interno, si sono considerati:

- limiti a carico (altezza piezometrica) imposto (condizione del primo tipo/Dirichlet "Hydraulic Head BC"), assegnato puntualmente in pozzi ai margini del modello di simulazione ed interpolati linearmente, per ottenere una distribuzione continua (Fig. 13);

- livello idrometrico noto (condizione del terzo tipo/ Cauchy "Fluid Transfer BC") lungo corsi d'acqua rilevanti (Fig .13); tale condizione permette di abbinare alle celle di calcolo il carico idraulico di riferimento con la conduttanza del mezzo ("transfer rate"); si applica a fiumi o laghi con una connessione limitata al sistema acquifero. L'afflusso / deflusso viene calcolato dall' equazione:

$$
\mathrm{Q}=\mathrm{A} \Phi\left(\mathrm{h}_{\mathrm{ref}}-\mathrm{h}\right)
$$

dove: Q: portata di scambio da / verso l'acquifero; A: superficie di riferimento; $\Phi$ : conduttanza; $\mathrm{h}_{\text {ref }}$ livello idrometrico; h: livello piezometrico.

- la ricarica verticale è assegnata al primo strato di calcolo ed è spazializzata distinguendo aree a ricarica crescente (Fig. 13 e 15);

- limiti laterali a "zero flusso" (disposti lungo le linee di flusso, ortogonalmente alle isopieze).

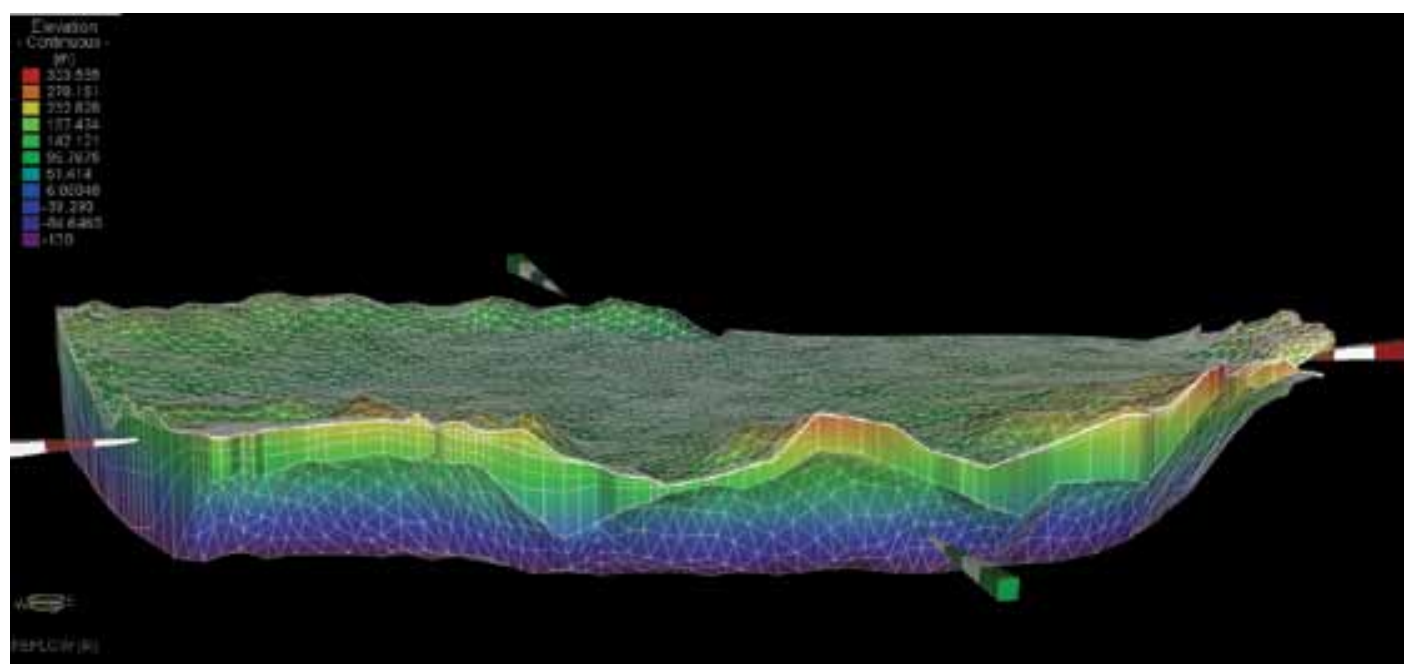

Fig. 12 - Griglia di calcolo tridimensionale.

Fig. 12 - 3D mesh.

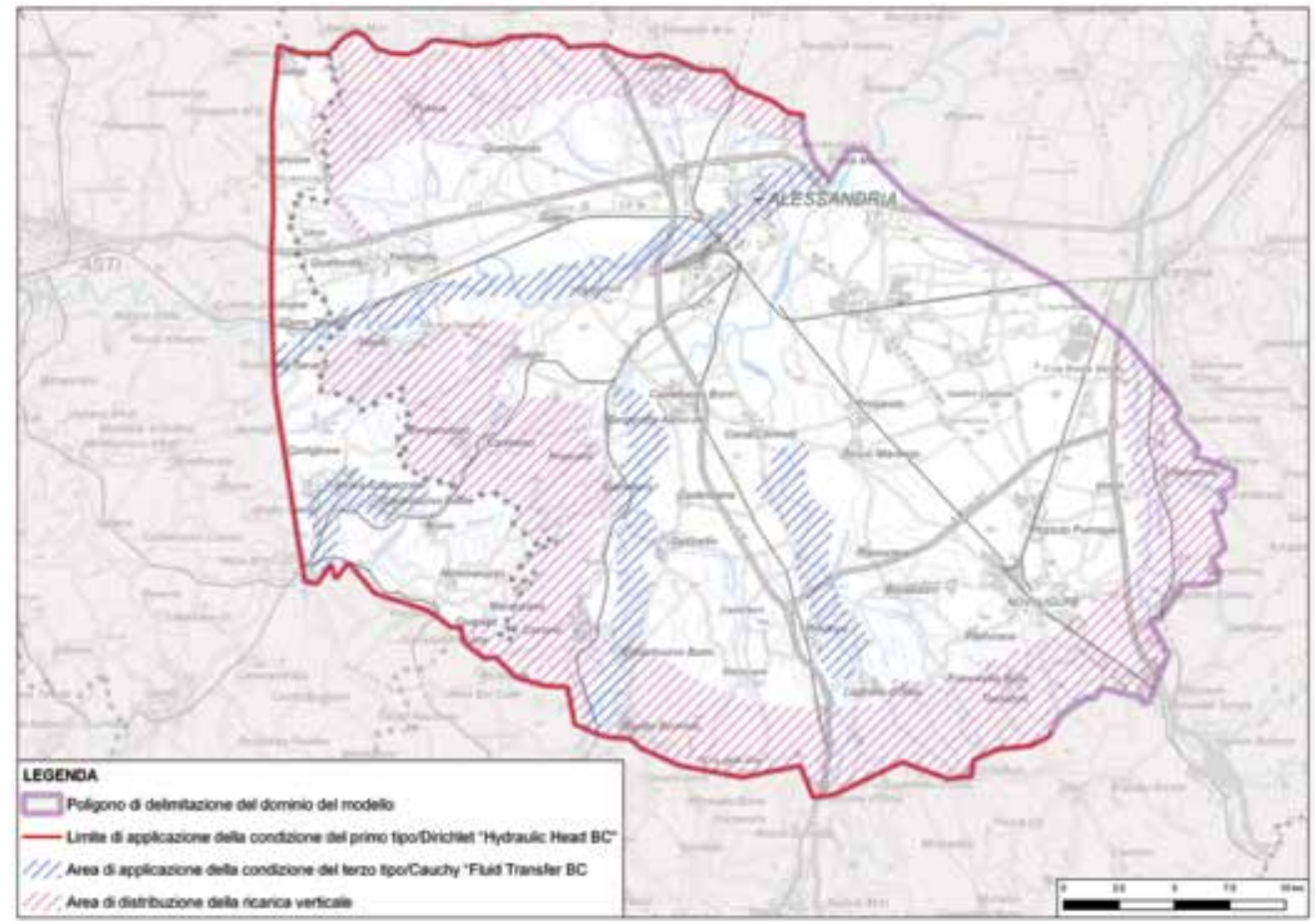

Fig. 13 - Distribuzione delle condizioni al contorno e all'interno.

Fig. 13 - Boundary ed internal conditions distribution. 


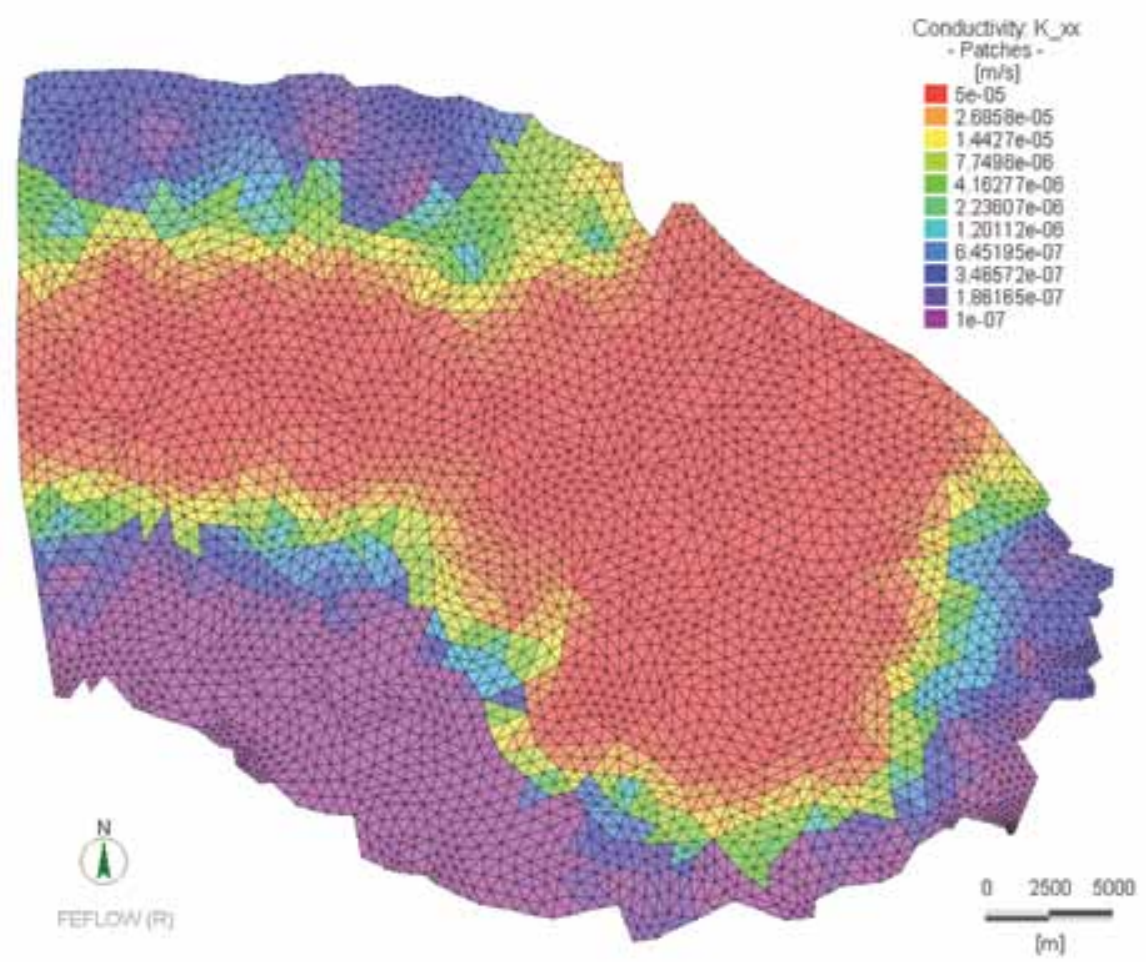

Fig. 14 - Distribuzione orizzontale delle Kx,Ky nell'unità E.

Fig. 14 - areal distribution of $K x, K y$ in $E$ unit.

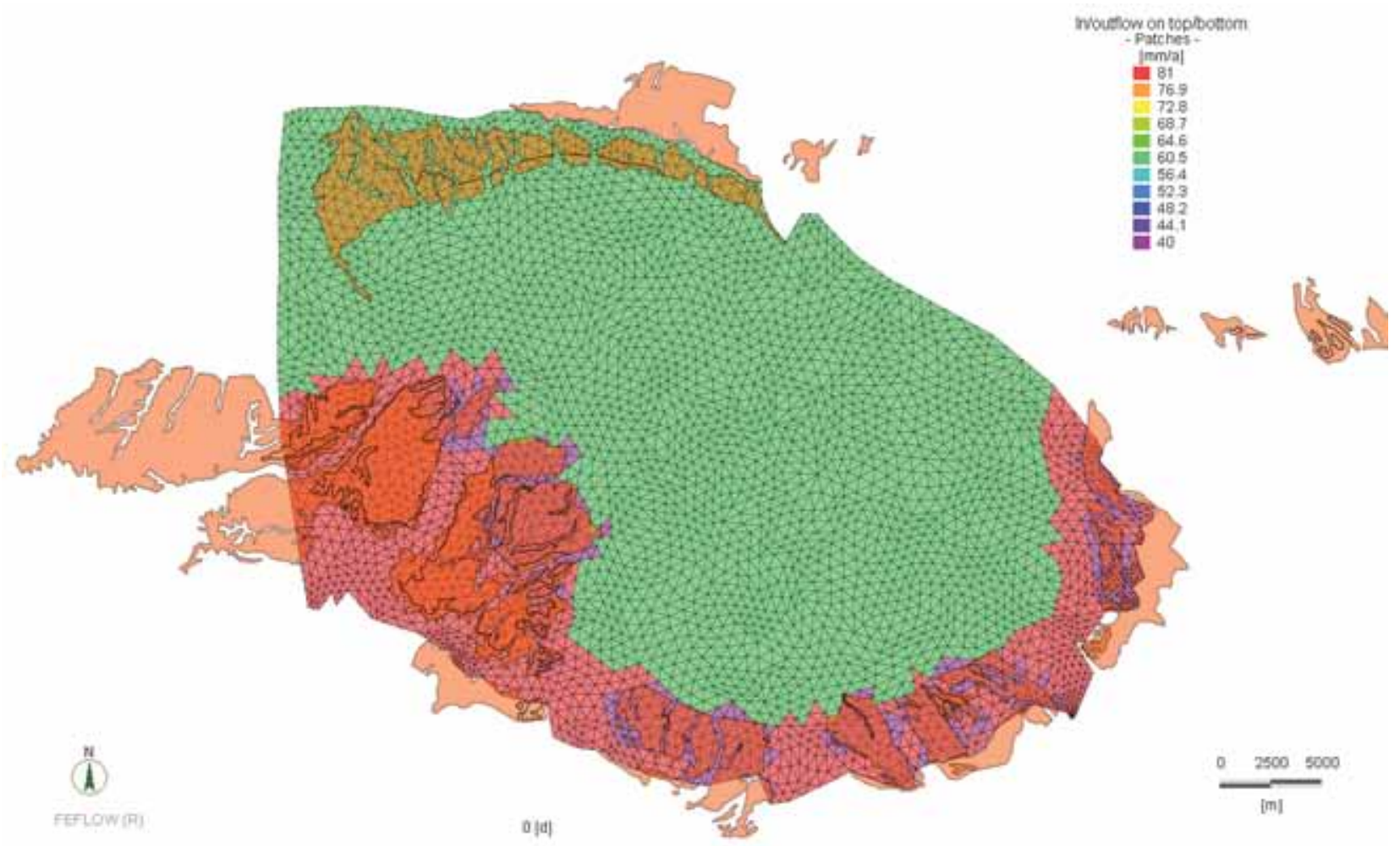

Fig. 15 - Distribuzione della ricarica verticale .

Fig. 15 - Zoning of vertical recharge. 
Il modello, calibrato in regime stazionario (in carenza di registrazioni piezometriche pluriennali in falda profonda), stima un errore medio di $1.19 \mathrm{~m}$ tra dati simulati ed osservati in pozzi selezionati (Fig. 17), compatibile con i margini di approssimazione della scala in esame, previa analisi di sensitività svolta con metodo "trial and error" riferita alla conducibilità idraulica orizzontale e verticale dell'acquifero profondo (Fig. 16). Per la piezometria di riferimento iniziale del modello di calcolo, sono interpolati i livelli misurati in pozzi filtranti selettivamente l'acquifero profondo.

Il modello di flusso è utilizzato a fini previsionali, per la revisione delle Aree di Riserva (RISE), nelle zone del Molinetto, in località Spinetta Marengo (tra 80-190 metri di profondità), e del campo pozzi di Predosa (tra 70 a 140 metri di profondità), dove il "Piano di Azione per la Messa in Sicurezza del Sistema di Approvvigionamento dell'ATO6" (ottobre 2017) prevede il potenziamento nello sfruttamento della risorsa idrica sotterranea e un'interconnessione infrastrutturaleacquedottistica tra $\mathrm{i}$ due poli di prelievo (European Commission, 2006). Per la delineazione delle RISE, è stata predisposta dapprima la modellazione del flusso e trasporto in falda advettivo (maggiormente cautelativo), quindi advettivodispersivo, più realistico e fisicamente basato. A tal fine il modello è utilizzato attivando la modalità di trasporto legato all'età, calcolando la LTE (Life-Time Expectancy, Cornaton et alii, 2011) - (tempo di vita atteso) ipotizzata a 10 e 25 anni "risalendo" i percorsi di flusso in arrivo ad un sistema di pozzi di prelievo (condizione al contorno LTE $=0$ ), per soddisfare il fabbisogno idrico di progetto previsto dall'EGATO in $0.4 \mathrm{~m}^{3} / \mathrm{s}$ nella RISE settentrionale (Spinetta Marengo - Molinetto) e $0.3 \mathrm{~m}^{3} / \mathrm{s}$ nella RISE meridionale (Predosa) In figura 18 vengono mostrati i risultati della modellazione advettiva (particletracking) in colore blu e advettivo-dispersiva in colore rosso.

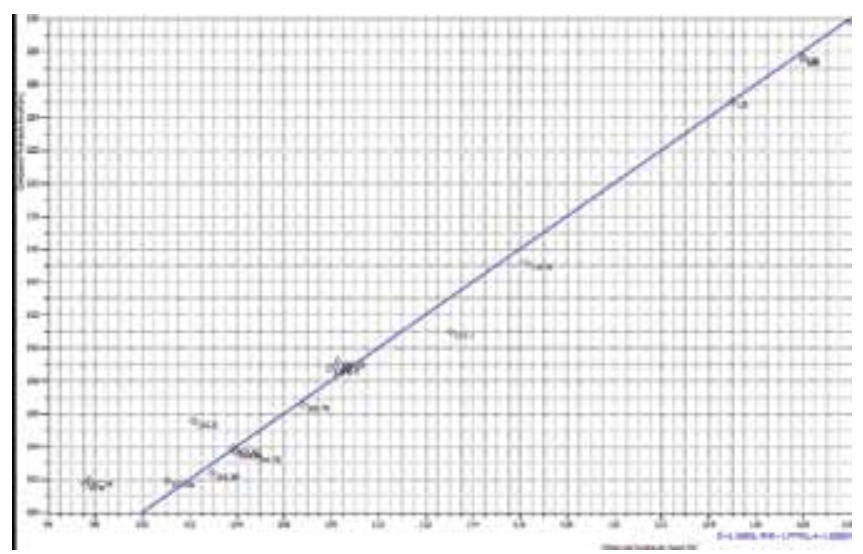

Fig. 17 - Correlazione fra dati piezometrici reali (ascisse) e simulati (ordinate).

Fig. 17 - Correlation line between real piezometric data (abscissa axis) and simulated data (ordinate axis).

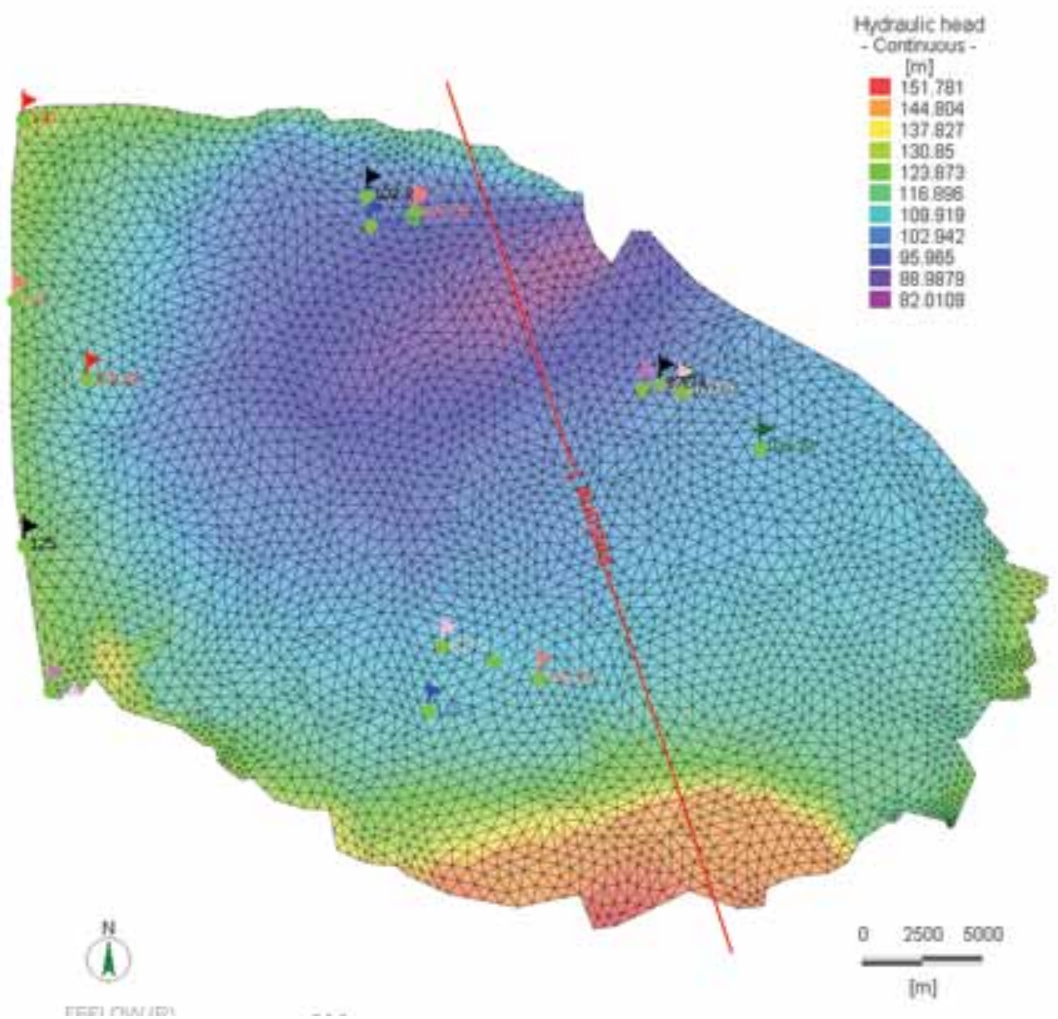

Fig. 16 - Ricostruzione bidimensionale della superficie piezometrica iniziale nel secondo strato di calcolo, con indicazione dei punti di calibrazione e della traccia di una sezione.

Fig. 16 - Reconstruction of the initial piezometric surface in the model (second layer), in 2D configuration, with calibration points and section track. 


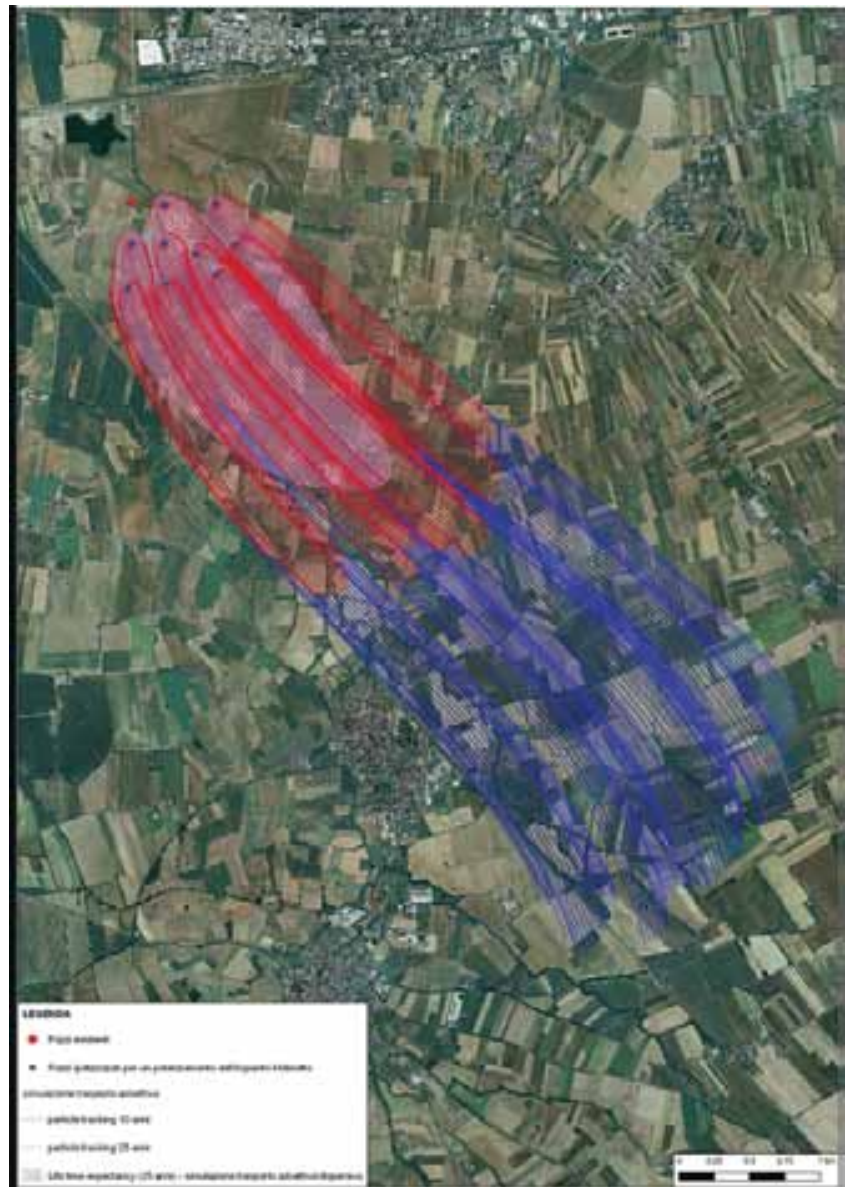

Fig. 18 - Risultati della modellazione advettiva (particle-tracking) e advettivodispersiva per l'ipotesi di RISE del Molinetto.

Fig. 18 - Results of advective modeling (particle-tracking) and advective-dispersive for the RISE Molinetto bypothesis.

\section{Risultati e discussioni}

Lanalisi del campo di flusso idrodinamico del sistema acquifero profondo, ricostruito tramite campagne di indagine piezometrica in pozzi selettivamente filtrati tra 80-300 metri da p.c., ha posto in evidenza la compartimentazione idrodinamica degli acquiferi in pressione rispetto alla restante pianura padana, per effetto del controllo strutturale profondo, esercitato dalla platea di depositi terziari sepolti, denominata "Struttura Tortona-Montecastello" (Irace et al. 2009).

Risulta ben delineata la distribuzione areale delle aree a gradiente idraulico verticale negativo (ai bordi collinari del sistema acquifero e nel settore apicale del conoide del T. Scrivia), positivo e con acqua risaliente verso la zona centrale (De Luca et al. 2019)

Lo screening dei parametri idrochimici, che ha identificato una dominante facies bicarbonato-calcico-magnesiaca, unitamente all'analisi dei principali rapporti ionici, ha consentito di individuare una zona di miscelazione $e$ diluizione centrale, opposta ai settori pedecollinari con grado di mineralizzazione più elevato, a controllo litologico specifico (es: depositi evaporitici).

La distribuzione areale del contenuto in isotopi stabili nei pozzi profondi è controllata dall'impronta isotopica dei bacini alimentanti gli acquiferi (impronta "alpino-tipo" nel distretto idrografico Tanaro-Bormida e appenninica nel distretto OrbaLemme-Scrivia).

Le datazioni delle acque hanno permesso di individuare una differenziazione regionale dei circuiti nel sistema idrogeologico, rilevando acque di età Olocenica-attuale in prossimità delle aree di ricarica e Pleistocenica media (Wurmiana) nel settore depocentrale del bacino, e valutare di conseguenza il grado di confinamento degli acquiferi profondi (laddove la variazione stagionale di composizione

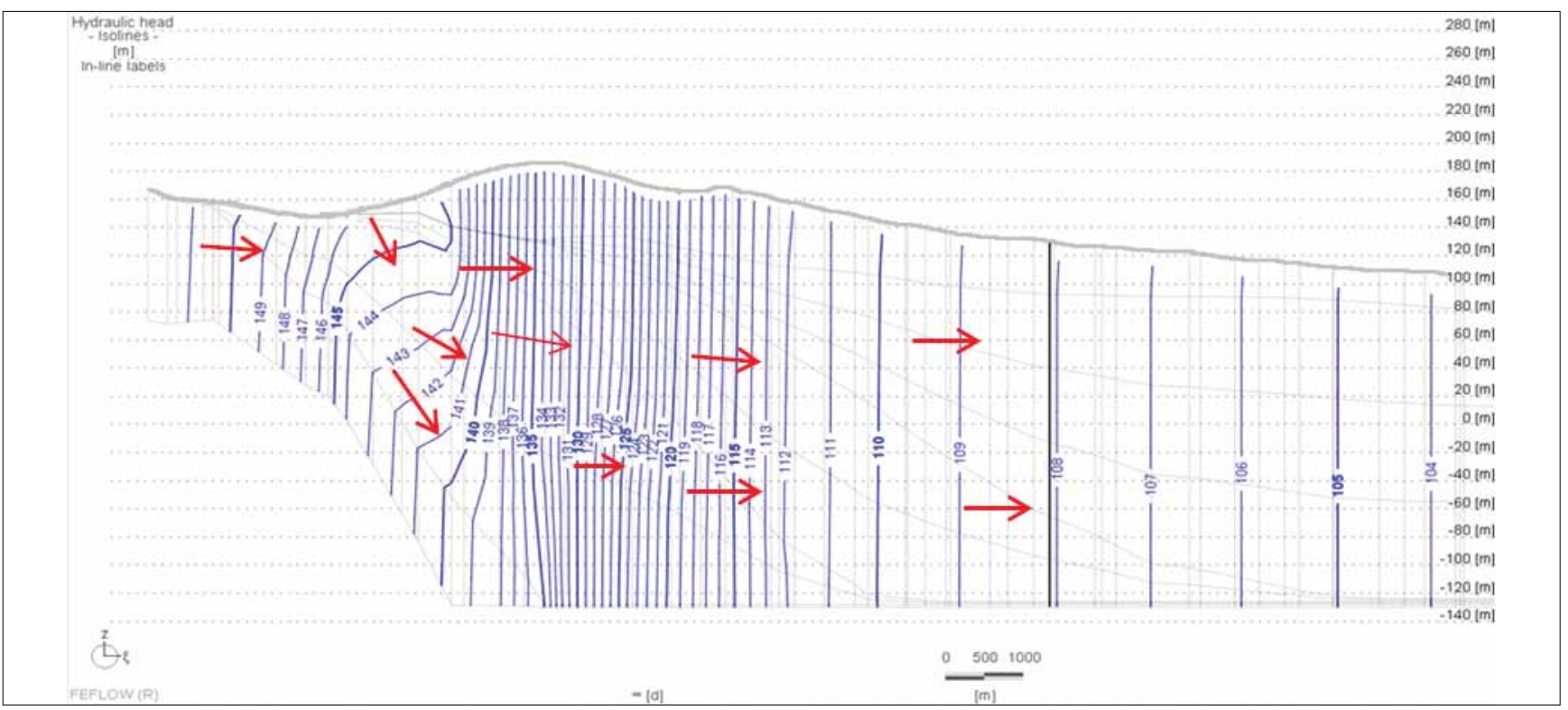

Fig. 19 - Sezione idraulica estesa dalle aree di ricarica alla zona depocentrale del bacino.

Fig. 19 - Hydraulic section from recharge areas to basin depocentre. 
in isotopi stabili è trascurabile) e la necessità di protezione degli stessi. La metodologia di datazione applicata potrà essere estesa ad un più ampio numero di punti di campionamento rispetto al set sperimentale sinora individuato, anche a supporto della calibrazione del campo di velocità di deflusso idrico sotterraneo ricostruito con il modello numerico di simulazione. Le aree di ricarica (De Luca et alii 2016) sono state delineate mediante valutazioni di carattere stratigrafico ed idrodinamico (gradiente idraulico verticale), attraverso il supporto del modello matematico di flusso.

\section{Conclusioni}

Sono state proposte opzioni di tutela integrata del sistema acquifero profondo, a protezione dell'approvvigionamento idropotabile del bacino alessandrino, mediante la definizione di aree di ricarica, aventi dimensioni complessive di $395 \mathrm{~km}^{2}$ e di zone di riserva (RISE), con superficie pari a $11 \mathrm{~km}^{2}$.

Le aree di ricarica (rappresentate in azzurro in figura 20), e quelle di riserva, (in arancione) sono state delimiate applicando tecniche idrogeologiche tradizionali (stratigrafiche, idrochimiche, petrofisiche, piezometriche, idrologiche), affiancate a determinazioni della variazione stagionale degli isotopi stabili dell'acqua e alla datazione delle acque circolanti in acquiferi semiconfinati-confinati tramite traccianti antropogenici e radioattivi

Lapproccio combinato di questi metodi con un modello di simulazione matematica permette di delineare un quadro affidabile in ordine alla stratificazione del sistema di flusso idrico profondo regionale e ai meccanismi di miscelazione tra gruppi acquiferi sovrapposti: le evidenze idrochimiche, di datazione delle acque e di prima stima delle velocità di flusso suggeriscono la probabile stratificazione di sistemi idrici confinati profondi a ricarica tardo-Pleistocenica, seguiti verso la superficie da sistemi multifalda semi-confinati, con ricarica da Olocenica sino a recente-attuale, attiva al limite di monte del bacino idrogeologico. In questo contesto, il fabbisogno idrico futuro previsto dall'Ente di Gestione in circa $0.7 \mathrm{~m}^{3} / \mathrm{s}$ potrà essere soddisfatto nelle RISE individuate in base a LTE di 10-25 anni, con un approccio sensibilmente cautelativo rispetto alla normativa vigente (aree di salvaguardia definite da linee isocrone a 180 e $60 \mathrm{gg}$ intorno ai campi-pozzi).

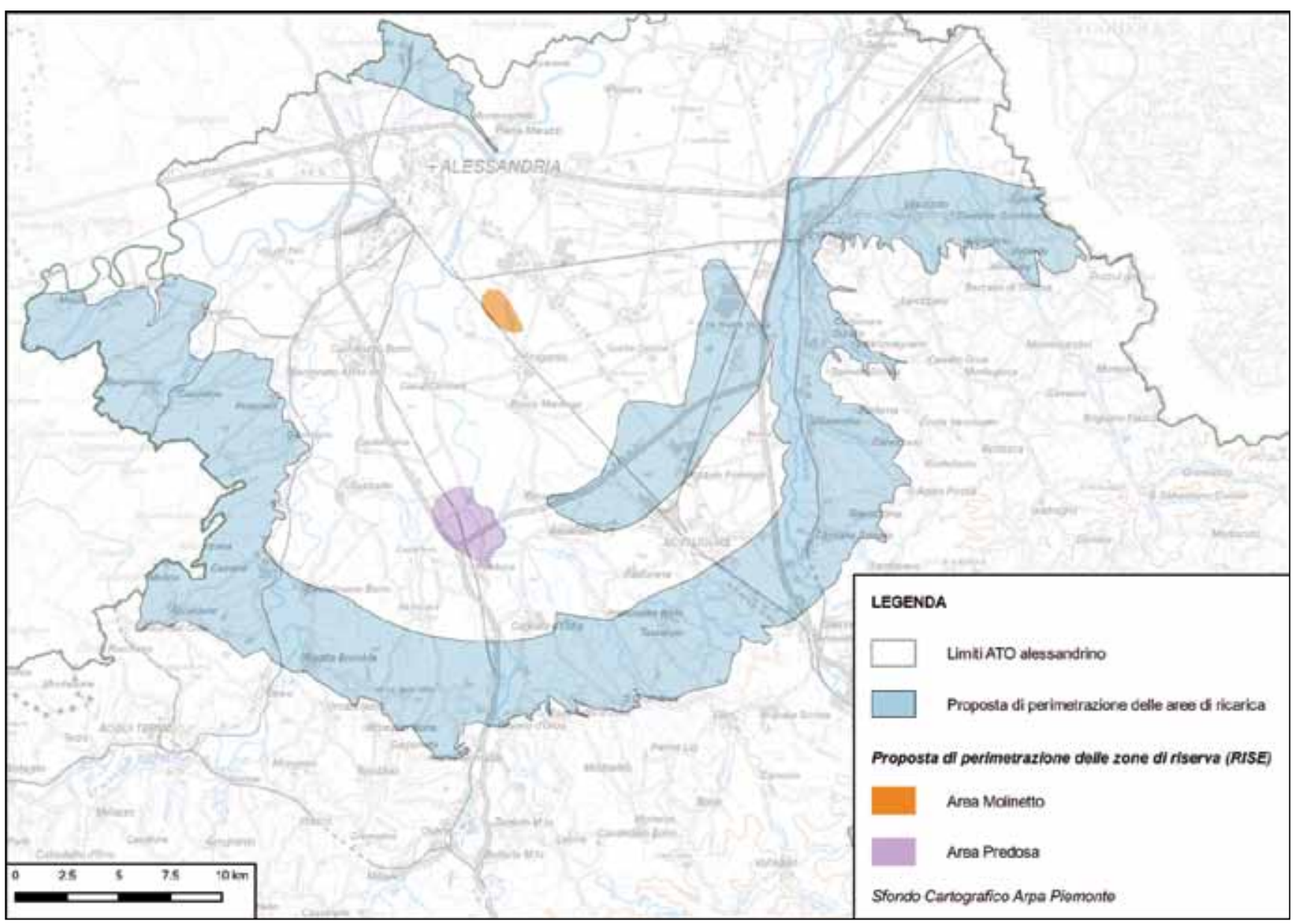

Fig. 20 - Proposta di delimitazione delle aree di ricarica e delle zone di riserva .

Fig. 20 - Proposal of delineation of recharge zones and reserve areas. 


\section{BIBLIOGRAFIA}

Bove A, Casaccio D, Destefanis E, De Luca DA, Lasagna M, Masciocco L, Ossella L, Tonussi M (2005) Criteri per l'individuazione delle principali zone di riserva e prima individuazione nella Regione Piemonte "Criteria for delineation of groundwater reserve area and first application in Regione Piemonte, IT". Idrogeologia della pianura piemontese, Chapter: 7, Publisher: Mariogros Industrie Grafiche S.p.A., Editors: Regione Piemonte, pp. 8

Braga G, Casnedi R (1976) I depositi alluvionali dello Scrivia (Provincia di Alessandria) "Alluvial deposits of Scriva River (Alessandria District)". Istituto di Ricerca sulle Acque. Quaderni dell'Istituto di Ricerca sulle Acque, C.N.R. - I.R.S.A. 281 e 2, 51

Cornaton F, Perrochet P (2011) Groundwater age, life expectancy and transit time distributions in advective-dispersive systems: 1. Generalized Reservoir Theory. Advanced in Water Resources arXiv: $1108.5483 \mathrm{v} 1$

Cruz-San Julian J, Araguas L, Rozanski K, Benavente J, Cardenale J, Hidalgo MC, Garcia-Lopezz S, Martinez-Garrido JC, Moral F, Olias M (1992) Sources of precipitation over South-Eastern Spain and groundwater recharge. An isotopic study. https://doi. org/10.1034/j.1600-0889.1992.t01-2-00005.x

De Luca, DA, Destefanis E, Falco M, Gisolo A, Governa M, Lasagna M, Petricig M (2016) Aggiornamento e revisione delle aree di ricarica degli acquiferi profondi nelle aree di pianura della Regione Piemonte "Upgrade and review of deep aquifer recharge zones in Regione Piemonte plain". (http://www.regione.piemonte.it/ambiente/acqua/ dwd/documentazione/ DD_268_21_07_2016_all_1.pdf).
De Luca D, Lasagna M, Mancini S (2019) Strategies for deep aquifers protection at local and regional scale: the Piedmont region example Geoingegneria Ambientale e Mineraria April 2019; Vol 1:24-29.

DHI-WASY: FEFLOW finite element subsurface flow and transport simulation system (2012) User's manual/reference manual/White papers. V. 6.1 . Technical report, DHI - Wasy GmbH, Berlin. www.feflow.com

European Commission (2006) Directive2006/118/EC of the European parliament and of the council of 12 December 2006 on the protection of groundwater against pollution and deterioration. Off $\mathrm{J}$ Eur Union L 372: 19-31.

IAEA - International Atomic Energy Agency (2006) Use of Chlorofluorocarbons in Hydrology. ISBN 92-0-1000805-8

Irace A, Clemente P, Natalicchio M, Ossella L, Trenkwalder S, De Luca DA, Mosca P, Piana F, Polino R, Violanti D (2009) Geologia e idrostratigrafia profonda della Pianura Padana occidentale "Geology and deep hydro-stratigraphic pattern of West Po Plain". (C) La Nuova Lito Firenze 2009 ISBN 978-88-904554-0-7

Marini L, Bonaria V, Guidi M, Hunziker JC, Ottonello G, Vetuschi Zuccolini M (2000) Fluid geochemistry of the Acqui Terme-Visone geothermal area (Piemonte, Italy). Applied Geochemistry 15, 917 935

Rozanski K, Araguas-Araguas L, Gonfiantini R (1993) Isotope patterns in modern global precipitation. Geoph. Monog. 78, 1-35

Schoeller H (1962) Leseaux Souterraines. Mason and Cie. Paris 642p.

Stiff HA (1951) Interpretation of Chemical water analysis by means of pattern. Journal of petrol Technology Vol. 3 No 10 Pp 15-17 